\title{
Invasão de ligustro no sub-bosque de um remanescente de floresta com araucária: uma abordagem demográfica
}

\author{
Privet invasion in the understory of an araucaria forest remnant: a demographic \\ approach
}

\begin{abstract}
Amanda da Silva Nunes ${ }^{\mathrm{I}}$, Pedro Higuchi" ${ }^{\mathrm{II}}$, Ana Carolina da Silva ${ }^{\mathrm{II}}$, Ricardo de Vargas Kilca'II, Mariele Alves Ferrer da Silva', Angélica Dalla Rosa ${ }^{\mathrm{IV}}$, Vanessa Fátima Soboleski', Juliana Pizutti Dallabrida ${ }^{\mathrm{I}}$, Karine Souza ${ }^{\mathrm{I}}$, Aline Pereira Cruz ${ }^{\mathrm{v}}$, Carla Luciane Lima ${ }^{\mathrm{I}}$, Lilian Iara Bet Stedille ${ }^{\mathrm{I}}$, Angela Camila Lemos ${ }^{\mathrm{VI}}$
\end{abstract}

\begin{abstract}
Resumo
Espécies invasoras têm ocasionado a perda de biodiversidade no mundo todo por ocuparem o lugar de nativas. O presente estudo objetivou avaliar a regeneração natural ao longo do tempo em um fragmento florestal invadido por Ligustrum lucidum W.T. Aiton e Ligustrum sinense Lour. no sul do Brasil, a fim de quantificar essa invasão. Para isso, foram alocadas cinco transecções em um fragmento de Floresta Ombrófila Mista em Lages (SC), sendo cada transecção dividida em parcelas com diferentes setores de distâncias da borda e em subparcelas para avaliação do componente regenerativo. Em 2012, os regenerantes de espécies arbóreas que se encontravam nas subparcelas foram identificados, plaqueteados e divididos nas seguintes classes: classe 1 - plantas com altura entre $10 \mathrm{~cm} \mathrm{e} 1 \mathrm{~m}$; classe 2 -plantas com altura entre 1 e $3 \mathrm{~m}$; classe 3 - plantas com altura maior que $3 \mathrm{~m}$ e diâmetro medido à altura do peito (DAP) menor que $5 \mathrm{~cm}$. Em 2014, esses indivíduos foram reavaliados, contabilizando os indivíduos mortos e recrutas e enquadrando os sobreviventes e recrutas nas atuais classes. Foram calculadas as taxas de mortalidade, recrutamento, mudanças de classe e mudança líquida. Os dados foram analisados por meio de uma análise de componentes principais (ACP) e tabela de contingência. Conclui-se que as espécies superaram a fase inicial do processo de invasão, demonstrando elevado incremento populacional na área estudada. Ainda, ficou evidenciado o particionamento espacial entre as espécies nativas e as exóticas, o que sugere a existência de competição interespecífica, cuja consequência pode ser a extinção local de representantes do grupo autóctone.
\end{abstract}

Palavras-chave: Dinâmica florestal; Espécies exóticas invasoras; Ligustro

Engenheira Florestal, Ma., Departamento de Engenharia Florestal, Universidade do Estado de Santa Catarina, Av. Luiz de Camões, 2090, CEP 88520-000, Lages (SC), Brasil. amandaeng.f@gmail.com (ORCID: 0000-0001-6669-3936) / mariele.ferrer@hotmail.com (ORCID: 0000-0003-2599-7356) / vanessasoboleski@hotmail.com (ORCID: 0000-0002-6817-2517) / juli_ajuri@hotmail.com (ORCID: 0000-0002-9766-8714) / karisouza@hotmail.com (ORCID: 0000-0002-0370-1502) / carla_engflorestal@yahoo.com.br (ORCID: 0000-0002-7943-3962) / lika_stedille@hotmail.com (ORCID: 0000-00034160-7709)

II Engenheiro Florestal, Dr., Professor do Departamento de Engenharia Florestal, Universidade do Estado de Santa Catarina, Av. Luiz de Camões, 2090, CEP 88520-000, Lages (SC), Brasil. higuchip@gmail.com (ORCID: 0000-0002-3855-555X) / carol_sil4@yahoo.com.br (ORCID: 0000-0002-1285640X)

III Biólogo, Dr., Departamento de Engenharia Florestal, Universidade do Estado de Santa Catarina, Av. Luiz de Camões, 2090, CEP 88520-000, Lages (SC), Brasil. ricardokilca@yahoo.com.br (ORCID: 0000-0003-1085-7641).

IV Graduanda em Engenharia Floresta, Departamento de Engenharia Florestal, Universidade do Estado de Santa Catarina, Av. Luiz de Camões, 2090, CEP 88520-000, Lages (SC), Brasil. angelica.dalla.rosa@hotmail.com (ORCID: 0000-0003-2359-5759)

Bióloga, Ma., Departamento de Engenharia Florestal, Universidade do Estado de Santa Catarina, Av. Luiz de Camões, 2090, CEP 88520-000, Lages (SC), Brasil. a.line_cruz@yahoo.com.br (ORCID: 0000-0002-9279-7293)

vı Bióloga, Ma., Programa de Pós-Graduação em Produção Vegetal, Universidade do Estado de Santa Catarina, Av. Luiz de Camões, 2090, CEP 88520000, Lages (SC), Brasil. angelacl@ymail.com (ORCID: 0000-0002-0170-0004) 


\begin{abstract}
The replacement of native species by invasive species has caused a worldwide loss of biodiversity. This study aimed to evaluate the natural regeneration over time in a forest fragment invaded by Ligustrum lucidum W.T. Aiton and Ligustrum sinense Lour. in southern Brazil, in order to quantify this invasion. To do so, five transects were allocated in a fragment of Araucaria Forest in Lages, Santa Catarina state, with each transect divided into plots in different categories of distance from the edge and into sub-plots for the evaluation of the regenerative component. In 2012, the regenerating tree species identified in the subplots were tagged and classified according to height classes: Class 1, plants with height between $10 \mathrm{~cm}$ and $1 \mathrm{~m}$; class 2, plants with height between 1 and $3 \mathrm{~m}$; and Class 3 , plants with a height greater than $3 \mathrm{~m}$ and DBH (diameter measured at breast height) less than $5 \mathrm{~cm}$. In 2014, these individuals were re-evaluated, counting the dead and recruited individuals and classifying the survivors and recruits into the existing height classes. Rates of mortality, recruitment, net change, and height class changes were calculated. The data were analyzed by a Principal Component Analysis (PCA) and contingency table. It was concluded that the species overcame the early stage of the invasion process, demonstrating great population growth in the study area. Also, a spatial partitioning between native and exotic species was observed, suggesting the existence of interspecific competition, the result of which may be the local extinction of species from the autochthonous group.
\end{abstract}

Keywords: Forest dynamics; Invasive exotic species; Privet tree

\title{
Introdução
}

A ação antrópica ocasiona múltiplos problemas nas comunidades vegetais, como o favorecimento da dispersão de espécies exóticas invasoras que, com isso, alcançam novos ambientes. A constante expansão da infraestrutura urbana agrava essa influência, pois ocasiona pressão na vegetação e na utilização dos recursos naturais em um ritmo mais veloz que a capacidade de regeneração da floresta (MALTA; SOUZA; SOUZA, 2012).

Espécies exóticas com capacidade de invasão apresentam-se privilegiadas em relação às espécies nativas, fato relacionado à coevolução, visto que, por muitas vezes, não possuem predadores naturais, o que facilita seu rápido crescimento e fixação, possibilitando uma vantagem competitiva para o estabelecimento e o desenvolvimento destas (CORREIA et al., 2016). Dentre essas espécies, destaca-se as do gênero exótico Ligustrum, que apresentam elevada representatividade em algumas florestas do sul do Brasil, sendo considerado um invasor agressivo na região, especialmente na Floresta Ombrófila Mista, tendo sido relatado, em alguns casos, com elevada abundância (GUIDINI et al., 2014; MIELKE et al., 2015; RODRIGUES et al., 2015).

Esse gênero é originário da Ásia, possui elevada capacidade regenerativa, alta plasticidade, ocupando tanto o interior da floresta como áreas de borda (ARAGÓN et al., 2014) e seus frutos são consumidos por diversas espécies da avifauna (FERREIRA, 2014). Dentre as espécies do gênero, Ligustrum lucidum tem sido citada como uma das espécies arbóreas mais agressivas em algumas regiões. Na Argentina, onde essa espécie também é invasora, o estudo de Gavier-Pizarro et al. (2012) demonstrou que, em 23 anos, a área ocupada aumentou 50 vezes, passando de 50 para 2.500 ha.

Considerando os impactos ecológicos e econômicos da invasão biológica, esse tema tem despertado cada vez mais o interesse de pesquisadores, que buscam informações básicas que possam subsidiar estratégias de controle. Nesse sentido, o estudo sobre a demografia de espécies florestais, especialmente no estrato regenerativo, pode fornecer informações relevantes sobre o processo de estabelecimento e desenvolvimento de espécies exóticas em ambientes naturais e o impacto destas sobre a comunidade biológica residente. Com isso, avaliar a dinâmica da regeneração natural permite interpretar como se desenvolve a vegetação ao longo do tempo, tornando-se uma ferramenta importante para o manejo e a conservação desses recursos.

Assim, o presente estudo objetivou avaliar a regeneração natural ao longo do tempo em um fragmento florestal invadido por Ligustrum lucidum e Ligustrum sinense no sul do Brasil, a fim de 
contextualizar a demografia desse gênero invasor em relação à comunidade nativa. Considerando que o processo de invasão não ocorre com a mesma intensidade ao longo do tempo, existindo uma fase inicial, denominada Lag, com pequeno ou nenhum incremento populacional, seguida por uma fase de intenso incremento (AIKIO; DUNCAN; HULME, 2010), buscou-se identificar em qual estágio do processo de invasão encontra-se Ligustrum spp. A hipótese testada foi de que esse gênero apresente elevado potencial invasor na área, de forma que sua representatividade no componente regenerativo tenha aumentado. Essa investigação permite gerar subsídios para o desenvolvimento de ferramentas de manejo e controle de um dos principais problemas ambientais da atualidade.

\section{Material e métodos}

O estudo foi realizado em um fragmento florestal localizado na propriedade da Empresa de Pesquisa Agropecuária e Extensão Rural de Santa Catarina (EPAGRI), com aproximadamente 11 ha, situado no município de Lages, planalto sul-catarinense (Figura 1), nas coordenadas $27^{\circ} 48^{\prime} 24,22$ ”'S e $50^{\circ} 20^{\prime} 06,97^{\prime \prime} O$. O fragmento, com vegetação classificada como Floresta Ombrófila Mista Montana, segundo o IBGE (2012), localiza-se na área urbana de Lages, em que Ligustrum lucidum é amplamente utilizado na arborização. O clima predominante na região é do tipo $\mathrm{Cfb}$, segundo a classificação de Köppen. De acordo com a estação meteorológica de Lages, administrada pela Empresa de Pesquisa Agropecuária e Extensão Rural de Santa Catarina (Epagri)/Centro de Informações de Recursos Ambientais e de Hidrometeorologia de Santa Catarina (Ciram)/Instituto Nacional de Meteorologia (INME), a precipitação e a temperatura média anual (1970-2010) foram de 1.682,80 $\mathrm{mm}$ e $15,9^{\circ} \mathrm{C}$, respectivamente. Com altitude próxima a $950 \mathrm{~m}$, o município está inserido na Bacia do rio Uruguai, com topografia, em sua maior parte, suave-ondulada a ondulada.

\section{Figura 1 - Localização das transecções e parcelas em um fragmento florestal invadido por} Ligustrum lucidum e Ligustrum sinense no sul do Brasil

Figure 1 - Transections and plots location in the forest fragment invaded by Ligustrum lucidum and Ligustrum sinense in southern Brazil

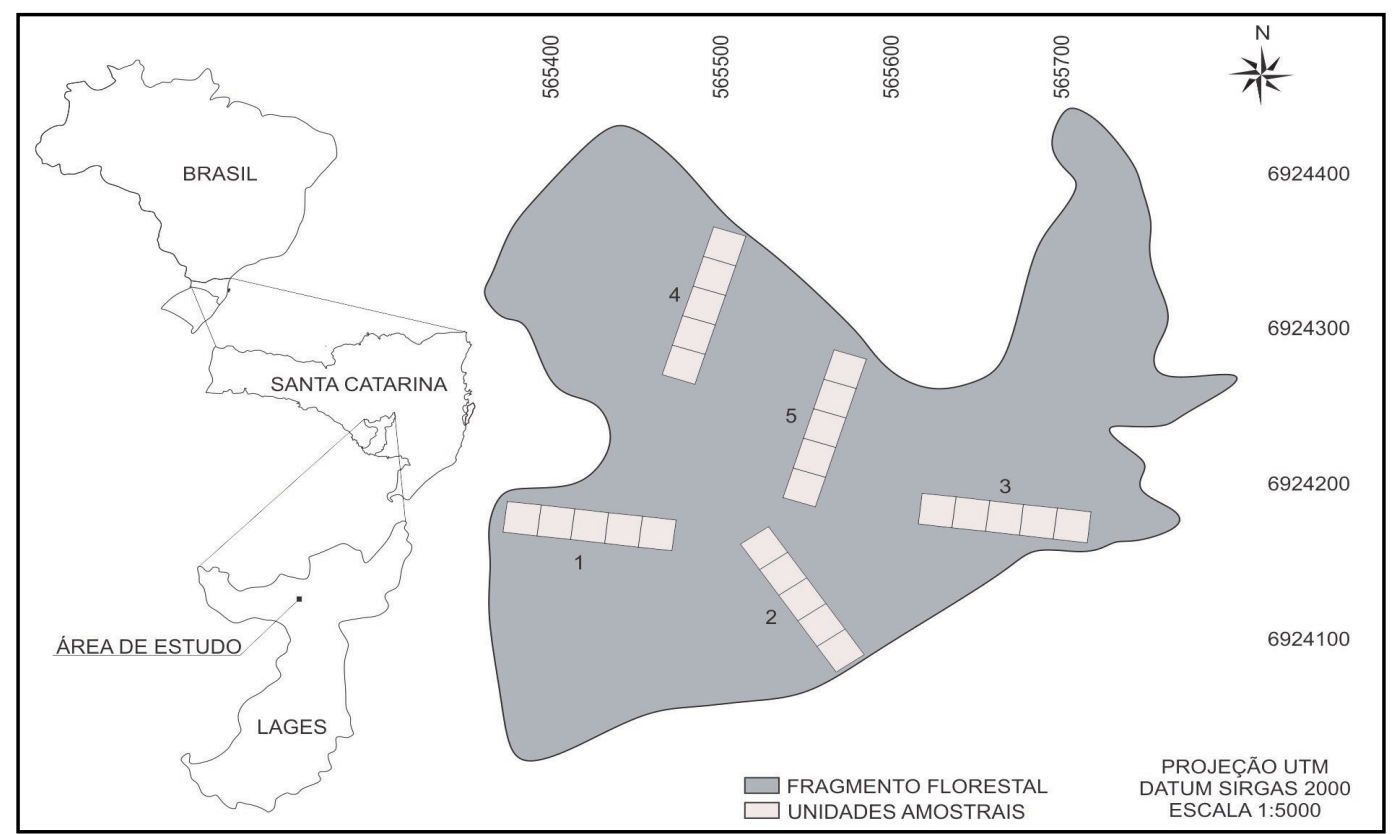

Fonte: Autores (2020) 
O estudo se deu em parcelas permanentes alocadas em 2012 (primeira avaliação), por Guidini et al. (2014). Dois anos após a primeira avaliação, em 2014, as mesmas parcelas foram novamente inventariadas (presente estudo), objetivando estudar a dinâmica do componente regenerativo e o avanço da invasão biológica na área. Em 2012, Guidini et al. (2014) alocaram cinco transecções permanentes de $20 \mathrm{~m}$ de largura por $100 \mathrm{~m}$ de comprimento, perpendiculares à borda da floresta, com distância mínima de $100 \mathrm{~m}$ entre si. Cada transecção foi dividida em cinco parcelas de $20 \times 20 \mathrm{~m}$, totalizando 25 parcelas de $400 \mathrm{~m}^{2}$, com diferentes setores de distâncias da borda. O primeiro setor, composto das primeiras parcelas de cada transecção, estava em contado com a borda, o segundo, a 20 a $40 \mathrm{~m}$ de distância da borda, o terceiro, a 40 a $60 \mathrm{~m}$, o quarto, a 60 a $80 \mathrm{~m}$, e o quinto, representado pelas últimas parcelas de cada transeção, a 80 a $100 \mathrm{~m}$ da borda. Para a avaliação do estrato regenerante arbóreo (indivíduos arbóreos com DAP menor que $5 \mathrm{~m}$ e altura maior que $10 \mathrm{~cm}$ ), as parcelas foram subdivididas em subparcelas com área variável de acordo com a classe dos regenerantes, seguindo-se a metodologia proposta por Volpato (1994), com adaptações: i) classe 1 - plantas com altura entre $10 \mathrm{~cm}$ e $1 \mathrm{~m}$, avaliadas em $5 \mathrm{~m}^{2}$; ii) classe 2 - plantas com altura entre 1 e $3 \mathrm{~m}$, avaliadas em $10 \mathrm{~m}^{2}$; iii) classe 3 - plantas com altura maior que $3 \mathrm{~m}$ e DAP menor que $5 \mathrm{~cm}$, avaliadas em $20 \mathrm{~m}^{2}$. Todos os indivíduos que se encontravam no interior das subparcelas foram identificados, marcados com plaquetas de alumínio e tiveram sua classe de altura registrada.

Em 2014, esses indivíduos foram novamente inventariados, sendo contabilizados os indivíduos mortos e os sobreviventes foram enquadrando nas atuais classes de altura. Indivíduos nas subparcelas que atingiram a altura de $10 \mathrm{~cm}$ foram considerados recrutas e inventariados segundo os mesmos critérios do primeiro inventário. A identificação das espécies, sempre que possível, foi realizada in loco ou os espécimes foram coletados para identificação posterior em laboratório com base em bibliografias especializadas, sendo os nomes científicos baseados na Lista de Espécies da Flora do Brasil (FORZZA et al., 2013) e na Flora Digital do Rio Grande do Sul (FLORA DIGITAL DO RIO GRANDE DO SUL, 2015), seguindo-se a classificação do APG IV (ANGIOSPERM PHYLOGENY GROUP, 2016).

Foram calculadas as taxas de mortalidade, recrutamento e de mudança de classe e mudança líquida em número de indivíduos. Os cálculos se basearam nos modelos algébricos adaptados de Sheil e May (1996):

$$
\begin{aligned}
& M=\left(1-\frac{N o-m}{N o}\right)^{1 / t} \times 100 ; \\
& C S=\left(1-\frac{N o-N C S}{N o}\right)^{1 / t} \times 100 ; \\
& M L=\left(\left(\frac{N_{t}}{N_{0}}\right)-1\right) \times 100 .
\end{aligned}
$$

$$
\begin{aligned}
& \mathrm{R}=\left(1-\left(1-\frac{\mathrm{r}}{\mathrm{Nt}}\right)\right)^{1 / \mathrm{t}} \times 100 ; \\
& \mathrm{CI}=\left(1-\left(\frac{\mathrm{No}-\mathrm{NCI}}{\mathrm{No}}\right)^{1 / \mathrm{t}} \times 100 ;\right.
\end{aligned}
$$

em que: $\mathrm{M}=$ taxa de mortalidade anual; $\mathrm{No}=$ número inicial de árvores; $\mathrm{m}=$ número de árvores mortas; $\mathrm{t}=$ intervalo de tempo entre os inventários; $\mathrm{R}=$ taxa de recrutamento anual; $\mathrm{r}=$ número de árvores recrutadas; $\mathrm{Nt}=$ número final de árvores sobreviventes depois de $\mathrm{t} ; \mathrm{CS}=$ taxa de mudança de classe para classes superiores; $\mathrm{NCS}=$ número de indivíduos de subiram de classe; $\mathrm{CI}=$ taxa de mudança de classe para classes inferiores; $\mathrm{NCI}=$ número de indivíduos que desceram de classe; $\mathrm{ML}$ = mudança líquida em número de indivíduos.

Foi realizada uma ACP com as taxas demográficas das espécies (dez indivíduos ou mais em 2012) com o propósito de identificar agrupamentos de espécies com o mesmo padrão de dinâmica e contextualizar as espécies invasoras em relação às nativas. A identificação dos eixos da ACP que sintetizaram, de forma significativa os padrões demográficos, foi realizada por meio da análise de ScreePlot. A verificação da distribuição espacial de Ligustrum lucidum e Ligustrum sinense em função dos setores de distância da borda $(0,20,40,60$ e $80 \mathrm{~m})$ foi realizada por meio de um teste de qui-quadrado aplicado a uma tabela de contingência. Todas as análises foram realizadas no R (R CORE TEAM, 2015), com o pacote Vegan (OKSANEN et al., 2015). 


\section{Resultados e discussão}

Na primeira avaliação foram amostrados 654 indivíduos regenerantes arbóreos, enquanto, em 2014, observaram 794, havendo, portanto, um aumento expressivo na abundância no período de dois anos de avaliação (Tabela 1), na ordem de $21,43 \%$. Esse aumento sugere que o componente regenerante se encontra em fase de construção silvegenética inicial (MACHADO; OLIVEIRA FILHO, 2010), típico de florestas em recuperação pós-distúrbios, o que está de acordo com o histórico observado na área.

Tabela 1 - Espécies amostradas no estrato regenerante do componente arbóreo de um fragmento florestal invadido por Ligustrum lucidum e Ligustrum sinense no sul do Brasil, com seus respectivos números de indivíduos amostrados em 2012 e 2014, taxas de mortalidade e de recrutamento

Table 1 - Sampling species in regenerative tree component in a forest fragment invaded by Ligustrum lucidum and Ligustrum sinense, in southern Brazil, with their respective number of individuals sampled in 2012 and 2014, mortality and recruitment rates

\begin{tabular}{|c|c|c|c|c|c|}
\hline \multirow[t]{2}{*}{ Famílias } & \multirow[t]{2}{*}{ Espécies } & \multicolumn{2}{|c|}{$\begin{array}{l}\text { Número de } \\
\text { indivíduos }\end{array}$} & \multirow[t]{2}{*}{ Tx.M (\%) } & \multirow[t]{2}{*}{ Tx.R (\%) } \\
\hline & & 2012 & 2014 & & \\
\hline Anacardiaceae & Lithraea brasiliensis Marchand & 3 & 2 & 42,26 & 29,28 \\
\hline \multirow[t]{3}{*}{ Aquifoliaceae } & Ilex microdonta Reissek & - & 1 & - & 100,00 \\
\hline & Ilex paraguariensis A. St.-Hil. & 2 & 2 & - & - \\
\hline & Ilex theezans Mart. ex Reissek & 13 & 11 & 8,01 & - \\
\hline \multirow[t]{4}{*}{ Asteraceae } & Baccharis dentata (Vell.) G. M. Barroso & 1 & 1 & - & - \\
\hline & Dasyphyllum spinescens (Less.) Cabrera & 1 & - & 100,00 & - \\
\hline & Dasyphyllum brasiliense (Spreng.) Cabrera & 3 & 3 & 18,35 & 18,35 \\
\hline & Moquiniastrum polymorphum (Less.) G. Sancho & 1 & 1 & - & - \\
\hline Berberidaceae & Berberis laurina Billb. & 2 & 2 & - & - \\
\hline Bignoniaceae & Jacaranda puberula Cham. & 17 & 19 & 6,07 & 11,14 \\
\hline Canellaceae & Cinnamodendron dinisii Schwacke & 10 & 9 & 10,55 & 5,72 \\
\hline Celastraceae & Monteverdia dasyclada (Mart.) Biral & 41 & 45 & 11,65 & 15,67 \\
\hline Dicksoniaceae & Dicksonia sellowiana Hook. & 17 & 9 & 27,23 & - \\
\hline Erythroxylaceae & Erythroxylum deciduum A. St.-Hil. & 2 & - & 100,00 & - \\
\hline Escalloniaceae & Escallonia bifida Link. \& Otto & 1 & 2 & - & 29,28 \\
\hline \multirow[t]{2}{*}{ Euphorbiaceae } & Sebastiania brasiliensis Spreng. & 2 & 2 & - & - \\
\hline & Gymnanthes klotzschiana Müll. Arg. & 36 & 33 & 7,20 & 3,07 \\
\hline \multirow[t]{4}{*}{ Fabaceae } & Dalbergia frutescens (Vell.) Britton & 20 & 18 & 13,39 & 8,71 \\
\hline & Machaerium sp. & 2 & 2 & - & - \\
\hline & Mimosa scabrella Benth. & - & 1 & - & 100,00 \\
\hline & Fabaceae 1 & 1 & 1 & - & - \\
\hline
\end{tabular}


Nunes, A. S.; Higuchi, P.; Silva, A. C.; Kilca, R. V.; Silva, M. A. F.; Rosa, A. D.; Soboleski, V. F.; Dallabrida, J. P.; Souza, K.; Cruz, A. P.; Lima, C. L.; Stedille, L. I. B.; Lemos, A. C.

Tabela 1 - Continuação ...

Table 1 - Continuation ...

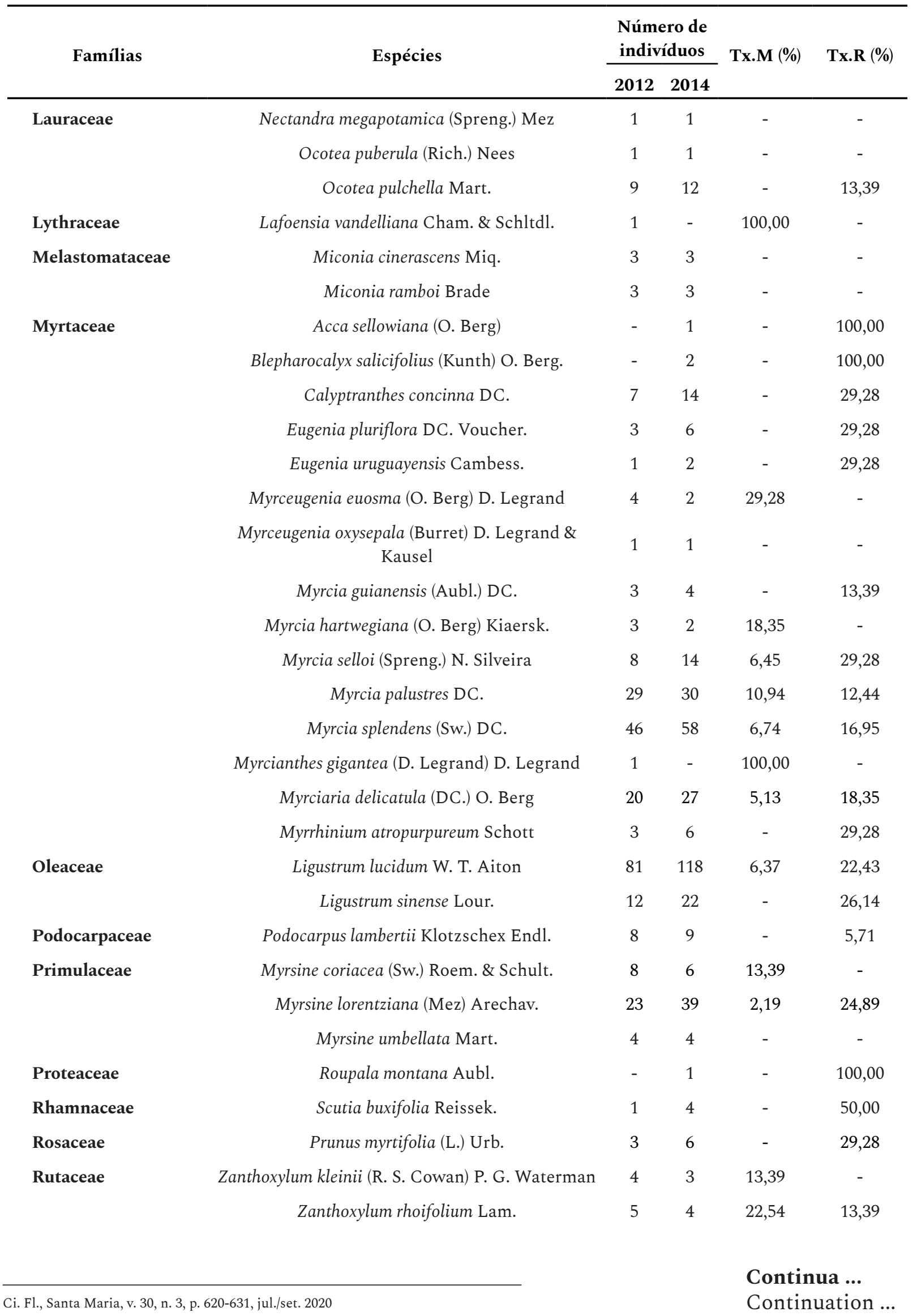


Tabela 1 - Conclusão ...

Table 1 - Conclusion ...

\begin{tabular}{|c|c|c|c|c|c|}
\hline \multirow[t]{2}{*}{ Famílias } & \multirow[t]{2}{*}{ Espécies } & \multicolumn{2}{|c|}{$\begin{array}{l}\text { Número de } \\
\text { indivíduos }\end{array}$} & \multirow[t]{2}{*}{ Tx.M (\%) } & \multirow[t]{2}{*}{ Tx.R (\%) } \\
\hline & & 2012 & 2014 & & \\
\hline \multirow[t]{4}{*}{ Salicaceae } & Banara tomentosa Clos & 63 & 76 & 5,71 & 14,16 \\
\hline & Casearia decandra Jacq. & 11 & 19 & - & 23,91 \\
\hline & Xylosma ciliatifolia (Clos) Eichler. & 3 & 5 & - & 22,54 \\
\hline & Xylosma tweediana (Clos) Eichler & 1 & 1 & - & - \\
\hline \multirow[t]{4}{*}{ Sapindaceae } & $\begin{array}{l}\text { Allophylus edulis (A. St.-Hil., Cambess. \& A. Juss.) } \\
\text { Radlk. }\end{array}$ & 17 & 21 & 2,98 & 12,71 \\
\hline & Allophylus guaraniticus (A. St.-Hil.) Radlk. & 8 & 9 & 6,45 & 11,80 \\
\hline & Cupania vernalis Cambess. & 1 & 1 & - & - \\
\hline & Matayba elaeagnoides Radlk. & 42 & 45 & 7,42 & 10,55 \\
\hline \multirow[t]{2}{*}{ Solanaceae } & Brunfelsia cuneifolia J. A. Schmidt & 3 & 2 & 18,35 & - \\
\hline & Solanum sanctaecatharinae Dunal & - & 2 & - & 100,00 \\
\hline Stryraceae & Styrax leprosus Hook. \& Arn. & 3 & 2 & 18,35 & - \\
\hline Symplocaceae & Symplocos uniflora (Pohl) Benth. & 1 & 1 & - & - \\
\hline Thymelaeaceae & Daphnopsis racemosa Griseb. & 22 & 31 & 2,29 & 17,69 \\
\hline Winteraceae & Drimys brasiliensis Miers. & 8 & 10 & 13,39 & 22,54 \\
\hline \multicolumn{2}{|c|}{ Total do grupo das invasoras (Ligustrum spp.) } & 93 & 140 & 5,53 & 23,00 \\
\hline \multicolumn{2}{|l|}{ Total de nativas } & 561 & 654 & 7,89 & 14,69 \\
\hline \multicolumn{2}{|l|}{ Total geral } & 654 & 794 & 7,55 & 16,03 \\
\hline
\end{tabular}

Fonte: Autores (2020)

Em que: Tx.M = taxa de mortalidade; Tx. $\mathrm{R}$ = taxa de recrutamento.

Em 2012, foram observadas 61 espécies, pertencentes a 45 gêneros e 29 famílias botânicas, enquanto, em 2014, esse número foi para 63 espécies, 47 gêneros de 28 famílias, representando um aumento de apenas 3,27\% na riqueza. Essa mudança ocorreu em razão do ingresso de seis novas espécies na comunidade regenerante (Ilex microdonta, Mimosa scabrella, Acca sellowiana, Blepharocalyx salicifolius, Roupala montana e Solanum sanctaecatharinae), de cinco gêneros (Mimosa, Acca, Blepharocalyx, Roupala e Solanum) e de uma família (Proteaceae) e do desaparecimento de quatro espécies (Dasyphyllum spinescens, Erythroxylum deciduum, Lafoensia vandelliana e Myrcianthes gigantea), de três gêneros (Erythroxylum, Lafoensia e Myrcianthes) e de duas famílias (Erythroxylaceae e Lythraceae). Destaca-se que todas as espécies citadas anteriormente apresentaram baixa abundância, ou seja, eram raras. Dessa forma, supõe-se que a baixa substituição florística observada seja o resultado de um ambiente de sub-bosque relativamente estável, tendendo ao estágio clímax, que não sofreu grandes alterações ambientais nem distúrbios durante o período avaliado, como a formação de grandes clareiras. Essa inferência se dá porque em condições de sub-bosque a dinâmica de clareiras é um dos principais fatores ecológicos condicionadores de variações florístico-estruturais ao longo do espaço e do tempo (VALLADARES et al., 2016).

Das espécies amostradas, duas são exóticas invasoras e ocorreram em elevada densidade: Ligustrum lucidum (81 indivíduos em 2012 e 118 em 2014, maior densidade na floresta) e Ligustrum 
sinescens Lour. (12 indivíduos em 2012 e 22 em 2014). Juntas, essas espécies foram responsáveis por $14,2 \%$ dos indivíduos na comunidade regenerante em 2012 e 17,6\% em 2014. De acordo com Theoharides e Duckes (2007), para ter sucesso, uma espécie invasora precisa superar diferentes etapas, iniciando-se pelo transporte até o local a ser invadido, passando pelos filtros abióticos para a colonização inicial, pelos filtros bióticos para o estabelecimento, até os filtros para a dispersão para outras áreas. No presente caso, considerando o aumento da participação relativa na comunidade regenerante e da observação no campo de indivíduos adultos reprodutivos, claramente as duas espécies de Ligustrum superaram os filtros abióticos e bióticos existentes para a colonização e o estabelecimento. Considerando que Ligustrum lucidum é amplamente visitada por aves frugíveras (FERREIRA, 2014), infere-se que L. sinense também seja, por apresentar fruto semelhante, e que ambas apresentem potencial de serem dispersas pela avifauna, de forma que ao longo do tempo se espera a dispersão para outros fragmentos na região, o que representa a última fase do processo de invasão (THEOHARIDES; DUCKES, 2007).

Ao se analisar as taxas demográficas das espécies, é possível entender melhor o potencial invasor de L. lucidum e $L$. sinense. Se forem consideradas apenas aquelas mais abundantes na comunidade (dez indivíduos ou mais em 2012), L. lucidum $\left(22,43 \%\right.$.ano $\left.{ }^{-1}\right)$ e L sinense $(26,14 \%$. ano $^{-1}$ ) estiveram, com Myrsine lorentziana (Mez) Arechav. $\left(24,89 \%\right.$.ano $\left.{ }^{-1}\right)$ e Casearia decandra Jacq. (23,91\%.ano-1), entre as com maiores taxas de recrutamento. Em relação à mortalidade, L. sinense se destacou como sendo a única sem indivíduos mortos e L. lucidum apresentou um valor relativamente baixo $\left(6,37 \%\right.$.ano $\left.{ }^{-1}\right)$, bem inferior, por exemplo, a Dicksonia sellowiana Hook. $\left(27,23 \%\right.$.ano $\left.{ }^{-1}\right)$ e Dalbergia frutescens (Vell.) Britton. $\left(13,39 \%\right.$.ano $\left.{ }^{-1}\right)$. Ainda, se forem consideradas em conjunto, observa-se que as espécies Ligustrum spp. apresentaram taxa de recrutamento $\left(23,00 \%\right.$. ano $\left.{ }^{-1}\right)$ e de mortalidade $\left(5,53 \%\right.$.ano $\left.{ }^{-1}\right)$, respectivamente, 1,6 vez maior $\left(14,69 \%\right.$.ano $\left.{ }^{-1}\right)$ e 1,4 vez menor $\left(7,89 \%\right.$.ano $\left.{ }^{-1}\right)$ do que as nativas.

A ordenação das populações em função de suas respectivas taxas demográficas (mortalidade, recrutamento, mudanças para classes superiores e inferiores e mudança líquida) também reforça o caráter invasor das espécies de Ligustrum (Figura 2). De acordo com a análise de ScreePlot (Figura 2a),apenas o eixo 1 da ordenação explicou uma parte significativa da inércia total dos dados (59,52\%). Esse eixo (Figura $2 \mathrm{~b}$ ) sintetizou um gradiente de padrões de dinâmica associado à capacidade de estabelecimento no fragmento. Espécies ordenadas mais à esquerda, onde se encontram $L$. lucidum e L. sinense, foram aquelas com elevada capacidade de estabelecimento, refletido pelo alto recrutamento, reduzida mortalidade, com subsequente incremento populacional (mudança líquida positiva), e elevado crescimento em altura, indicado pela taxa de mudança para classes superiores. Por outro lado, espécies ordenadas mais à direita foram aquelas com menos capacidade de se estabelecerem, pois apresentaram padrões demográficos opostos, sendo, assim, candidatas a uma eventual exclusão competitiva.

Os resultados aqui encontrados, com outros estudos já realizados (GUIDINI et al., 2014; MIELKE et al., 2015; RODRIGUES etal., 2015), reforçam a ideia de que es pécies do gênero Ligustrum realmente apresentam elevado potencial invasor na Floresta Ombrófila Mista do sul do Brasil, principalmente em florestas inseridas no ambiente urbano. Em um estudo no estado do Paraná, considerando o componente adulto (DAP $>5 \mathrm{~cm}$ ), Rodrigues et al. (2015) verificaram o mesmo padrão demográfico, com Ligustrum lucidum apresentando elevado incremento populacional em um período de quatro anos. Já em um estudo na Argentina, Aragón et al. (2014) destacaram a elevada capacidade competitiva em relação às nativas. Assim, fica evidenciada a vantagem competitiva de espécies de Ligustrum, refletida em suas taxas demográficas, em relação a maior parte das espécies nativas. Uma das principais hipóteses para explicar esse padrão sustenta que a ausência de inimigos naturais (e.g. herbívoros, patógenos) fora da área de ocorrência natural libera essas espécies de possíveis fatores controladores do tamanho populacional (KEANE; CRAWLEY, 2002). Assim, em razão do elevado potencial invasor identificado, presume-se que esse processo resulte em uma das consequências clássicas da invasão biológica, que inclui extinção local e perda da diversidade (GAERTNER et al., 2009), e, no presente caso, Dicksonia sellowiana e 
Dalbergia frutescens aparecem como candidatas a serem deslocadas de seus respectivos ambientes naturais.

Figura 2 - Screeplot (a) para o resultado da ACP (b), das taxas de dinâmica das espécies (dez indivíduos ou mais em 2012) do estrato regenerante em um fragmento florestal invadido por Ligustrum lucidum e Ligustrum sinense no Sul do Brasil. R: taxa de recrutamento $\left(\%\right.$.ano $\left.{ }^{-1}\right)$; M: taxa de mortalidade (\%.ano $\left.{ }^{-1}\right)$; ML: mudança líquida em número de indivíduos (\%.ano $\left.{ }^{-1}\right)$; CS: mudança para classes superiores $\left(\%\right.$ ano $\left.^{-1}\right)$; CI: mudança para classes inferiores $\left(\%\right.$.ano $\left.{ }^{-1}\right)$

Figure 2 - Scree plot (a) for the results of a Principal Component Analysis (PCA) (b), of dynamics rates of regenerative tree species ( $\geq$ ten individuals in 2012), in a forest fragment invaded by Ligustrum lucidum and Ligustrum sinense, in southern Brazil. $\mathrm{R}=$ recruitment rate $\left(\%\right.$.year $\left.{ }^{-1}\right) ; \mathrm{M}=$ mortality rate $\left(\%\right.$.year $\left.{ }^{-1}\right) ; \mathrm{ML}=$ number of individuals net change $\left(\%\right.$.year $\left.{ }^{-1}\right) ; \mathrm{CS}=$ upper classes change $\left(\%\right.$.year $\left.{ }^{-1}\right) ; \mathrm{CI}=$ lower classes change $\left(\%\right.$.year $\left.{ }^{-1}\right)$

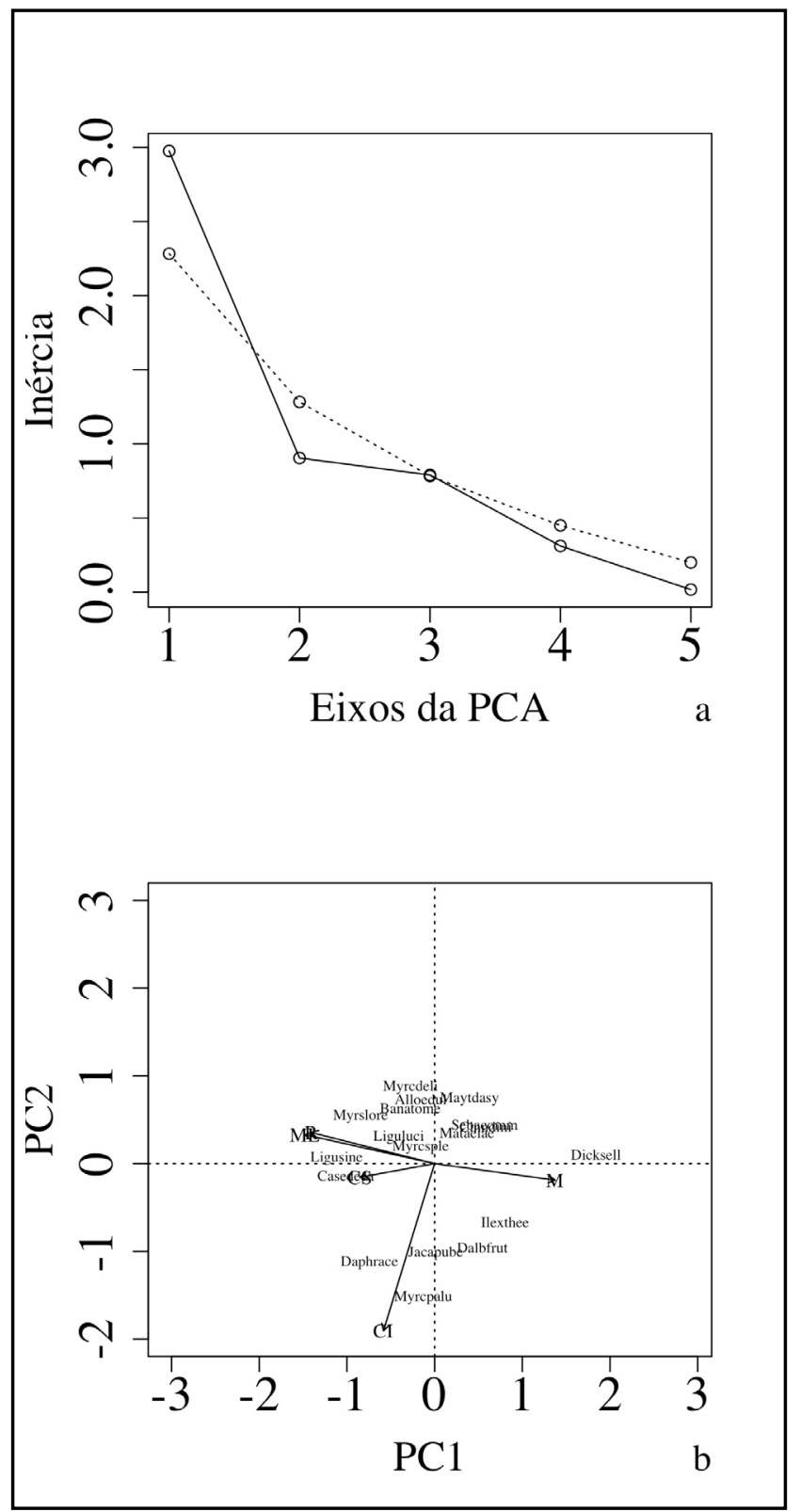

Fonte: Autores (2020) 
Quando se analisa a presença das espécies invasoras nos diferentes setores avaliados, é possível perceber que ocorrem de forma heterogênea no espaço, porém sem relação com a distância da borda (Tabela 2). Dentre os aspectos abióticos determinantes para a ocorrência de Ligustrum lucidum, Aguirre-Acosta, Kowaljow e Aguilar (2014) observaram maior presença de plântulas em solos mais úmidos, com maiores teores de carbono, matéria orgânica e nitrogênio. Rodrigues et al. (2015) destacaram a baixa tolerância à compactação do solo. Enquanto o gênero invasor ocorreu preferencialmente nos setores 2 ( $20 \mathrm{~m}$ da borda) e 3 ( $60 \mathrm{~m}$ da borda) em ambos os anos de avaliação, as nativas ocorreram preferencialmente nos setores 1,4 e 5 . Dessa forma, fica evidente que a regeneração natural no fragmento ocorre de forma espacialmente particionada entre nativas e invasoras, o que é um indicativo da existência de competição interespecífica, uma vez que a capacidade de espécies de Ligustrum inibir a sobrevivência e o crescimento de espécies autóctones já foi relatada por outros estudos (e.g. GREENE; BLOSSEY, 2012).

\section{Tabela 2 - Número de indivíduos pertencentes a espécies nativas e ao grupo de Ligustrum lucidum e Ligustrum sinense de 2012 e 2014, por setores de distância da borda, em um fragmento florestal no sul do Brasil}

Table 2 - Number of individuals belonging to native species and Ligustrum lucidumand Ligustrum sinense group in 2012 and 2014, by sectors of edge distance, in a forest fragment, in southern Brazil

\begin{tabular}{|c|c|c|c|c|}
\hline \multirow[b]{2}{*}{ Setores } & \multicolumn{2}{|c|}{2012} & \multicolumn{2}{|c|}{2014} \\
\hline & Nativas & Invasoras & Nativas & Invasoras \\
\hline 1 & $131(123,52)$ & $13(20,48)$ & $153(141,67)$ & $19(30,32)$ \\
\hline 2 & $89(108,08)$ & $37(17,91)$ & $100(136,73)$ & $66(29,27)$ \\
\hline 3 & $110(113,23)$ & $22(18,77)$ & $145(145,79)$ & $32(31,21)$ \\
\hline 4 & $116(111,51)$ & $14(18,49)$ & $136(124,38)$ & $15(26,62)$ \\
\hline 5 & $115(104,65)$ & $7(17,35)$ & $120(105,43)$ & $8(22,57)$ \\
\hline
\end{tabular}

Fonte: Autores (2020)

Em que: Valores entre parênteses representam o número de indivíduos esperado, de acordo com uma distribuição regular, pelo teste de qui-quadrado $\left(2012: \chi^{2}=35,99 ; p<0,01 ; 2014: \chi^{2}=78,70 ; p<0,01\right)$.

\section{Conclusões}

Em dois anos de avaliação, o componente regenerativo do fragmento avaliado caracterizouse por apresentar um incremento expressivo na abundância de indivíduos e estabilidade na riqueza. Nesse contexto, o gênero Ligustrum se destacou por sua expressiva participação na comunidade, que aumentou entre os anos de avaliação, o que demonstra que superou a fase inicial do processo de invasão. Ainda, ficou evidenciado o particionamento espacial entre as espécies nativas de Ligustrum, o que sugere a existência de uma relação negativa de inibição entre esses grupos, o que pode resultar em extinção local de espécies autóctones, como Dicskonia sellowiana e Dalbergia frutescens. Dessa forma, conclui-se que Ligustrum lucidum e Ligustrum sinense apresentaram elevado potencial invasor no fragmento de Floresta Ombrófila Mista avaliado. 


\section{Agradecimentos}

À Fundação de Amparo à Pesquisa e Inovação do Estado de Santa Catarina pela concessão de bolsa à primeira autora do trabalho e pelo apoio por meio do Programa de Apoio à Pesquisa/ Universidade do Estado de Santa Catarina. Ao Conselho Nacional de Desenvolvimento Científico e Tecnológico pela concessão de bolsa de produtividade para o segundo e terceiro autores. À Epagri/Lages por permitir a realização do estudo em área de sua propriedade.

\section{Referências}

AIKIO, S.; DUNCAN, R. P.; HULME, P. E. Lag-phases in alien plant invasions: separating the facts from the artefacts. Oikos, Hoboken, v. 119, n. 2, p. 370-378, 2010.

ANGIOSPERM PHYLOGENY GROUP IV. An update of the Angiosperm Phylogeny Group classification for the orders and families of flowering plants: APG IV. Botanical Journal of the Linnean Society, London, v. 181, n.1, p. 1-20, 2016.

AGUIRRE-ACOSTA, N.; KOWALJOW, E.; AGUILAR, R. Reproductive performance of the invasive tree Ligustrum lucidum in a subtropical dry forest: does habitat fragmentation boost or limit invasion? Biological Invasions, Nova York, v. 16, n. 7, p. 1397-1410, 2014.

ARAGÓN, R. et al. Exotic species as modifiers of ecosystem processes: litter decomposition in native and invaded secondary forests of NW Argentina. Acta Oecologica, Amsterdam, v. 54, p. 21-28, 2014.

CORREIA, M. et al. Evidence for enemy release and increased seed production and size for two invasive Australian acácias. Journal of Ecology, Londres, v. 104, n. 5, p. 1391-1399, 2016.

FERREIRA, R. C. Estrutura da guilda das aves frugíveras da APA Parque e Fazenda do Carmo, São Paulo, Brasil. Enciclopédia Biosfera, Goiânia, v. 10, n. 18, p. 3262-3279, 2014.

FLORA DIGITAL DO RIO GRANDE DO SUL. [Website]. Porto Alegre, 2015. Disponível em: http://www6.ufrgs.br/fitoecologia/florars. Acesso em: 14 out. 2015.

FORZZA, R. C. et al. Lista de Espécies da Flora do Brasil. Rio de Janeiro: Jardim Botânico do Rio de Janeiro, 2013. Disponível em: http://reflora.jbrj.gov.br/jabot/listaBrasil/ConsultaPublicaUC/ ConsultaPublicaUC.do. Acesso em: 15 out. 2015.

GAERTNER, M. et al. Impacts of alien plant invasions on species richness in Mediterraneantype ecosystems: a meta-analysis. Progress in Physical Geography, Thousand Oaks, v. 33, n. 3, p. 319-338, 2009.

GAVIER-PIZARRO, G. I. et al. Monitoring the invasion of an exotic tree (Ligustrum lucidum) from 1983 to 2006 with 1 Landsat TM/ETMp satellite data and support vector machines in Córdoba, Argentina. Remote Sensing of Environment, Amsterdam, v. 122, p. 134-145, 2012.

GREENE, B. T.; BLOSSEY, B. Lost in the weeds: Ligustrumsinense reduces native plant growth and survival. Biological invasions, Dordrecht, v. 14, n. 1, p. 139-150, 2012.

GUIDINI, A. L. et al. Invasão por Espécies Arbóreas Exóticas em Remanescentes Florestais no Planalto Sul Catarinense. Revista Árvore, Viçosa, MG, v. 38, n. 3, p. 469-478, 2014.

IBGE. Manual técnico da vegetação brasileira. Rio de Janeiro, 2012. 271 p.

KEANE, R. M.; CRAWLEY, M. J. Exotic plant invasions and the enemy release hypothesis. Trends in ecology \& evolution, Amsterdam, v. 17, n. 4, p. 164-170, 2002. 
MACHADO, E. L. M.; OLIVEIRA FILHO, A. T. Spatial patterns of tree community dynamics are detectable in a small (4 ha) and disturbed fragment of the Brazilian Atlantic forest. Acta Botanica Brasilica, Belo Horizonte, v. 24, n. 1, p. 250-261, 2010.

MALTA, J. A.; SOUZA, H. T.; SOUZA. R. M. Fitogeografia e regeneração natural em florestas urbanas de São Cristóvão/SE-Brasil. Boletín del Instituto de Geografía, Cidade do México, n. 77, p. 48-62, 2012.

MIELKE, E. C. et al. Espécies exóticas invasoras arbóreas no parque da barreirinha em Curitiba: registro e implicações. Ciência Florestal, Santa Maria, v. 25, n. 2, p. 327-336, 2015.

OKSANEN, J. et al. Vegan: communityecologypackage. [S. l.: s. n.], 2015. Disponível em: http:// cran.r-project.org/package=vegan. Acessoem: 30 jul. 2015.

R CORE TEAM. R: a language and environment for statistical computing. Viena: R Foundation for StatisticalComputing, 2015. Disponível em: http://www.r-project.org. Acesso em: 30 jul. 2015.

RODRIGUES, A. L. et al. Dinâmica e modelagem autologistica da distribuição da espécie invasora Ligustrum lucidum W.T. Aiton em floresta nativa. Scientia Forestalis, Piracicaba, v. 43, n. 107, p. 665-674, 2015.

SHEIL, D.; MAY, R. M. Mortality and recruitment rate evaluations in heterogeneous tropical forests. Journal of Ecology,Oxford, v. 84, n. 1, p. 91-100, 1996.

THEOHARIDES, K. A.; DUKES, J. S. Plant invasion across space and time: factors affecting nonindigenous species success during four stages of invasion. New Phytologist, Hoboken, v. 176, n. 2, p. 256-273, 2007.

VALLADARES, F. et al. Shedding light on shade: ecological perspectives of understorey plant life. Plant Ecology \& Diversity, Abingdon, v. 9, n. 3, p. 237-251, 2016.

VOLPATO, M. M. L. Regeneração natural em uma floresta secundária no domínio de Mata Atlântica: uma análise fitossociológica. 1994. Dissertação (Mestrado em Ciência Florestal) Universidade Federal de Viçosa, Viçosa, MG, 1994. 